\title{
Theoretical consideration of the growth morphology of crystals
}

\author{
Xiang-Yang Liu and P. Bennema \\ Research Institute for Materials, Laboratory of Solid State Chemistry, Faculty of Science, University of Nijmegen, Toernooiveld, \\ 6525 ED Nijmegen, The Netherlands
}

(Received 11 October 1994; revised manuscript received 4 October 1995)

\begin{abstract}
A theoretical approach for the prediction of the growth forms of crystals is presented on the basis of the kinetics of crystal growth. To describe the growth morphology of crystals, the more precise relations between the relative growth rate of the crystal faces $\{h k l\} R_{h k l}^{\text {red }}$ and habit-controlling factors are derived from the Burton-Cabrera-Frank (BCF) models, the Chernov model, and the two-dimensional crystal growth models. According to these established relations, the growth morphology of crystals is directly related to internal and external habit-controlling factors. Among these factors, the attachment energy $E_{h k l}^{\text {att }}$, the surface scaling factor $C_{l(h k l)}^{*}$ and the molecular orientational factor $t_{h k l}$ play an essential role in controlling the growth habit of crystals. As the key external habit-controlling factors, $C_{l(h k l)}^{*}$ and $t_{h k l}$ depend on the structure of the interface, the composition of the system, and other parameters affecting the growth process. Correspondingly, $E_{h k l}^{\text {att }}$ represents the influence of the crystal structure on the morphology of the crystal. To examine the validity of our formalisms, the morphology of paraffin crystals grown from $n$-alkane solutions and that of $\mathrm{C}_{60}$ crystals grown from the vapor are predicted. The results are in excellent agreement with observations. Within the framework of our approach, the relation $R_{h k l}^{\text {red }} \sim E_{h k l}^{\text {att }}$ proposed in the Hartman-Perdok theory can be obtained by simplifying our formulas. Finally, morphological changes resulting from different experimental conditions are generally discussed based on our formalisms.
\end{abstract}

\section{INTRODUCTION}

The prediction and control of the growth habits of crystals are nowadays becoming increasingly interesting to crystal growers and industries. For crystal growers, in order to obtain crystals with a sufficient size in a certain orientation, it is desirable to achieve a suitable growth habit. For industries, the growth habit of crystals plays a crucial role in industrial crystallization and quality control.

It has long been realized ${ }^{1-3}$ that the growth morphology of crystals is determined by the anisotropy of growth rates. A crystallographic orientation with a higher growth rate has less chance to appear in crystal form and may eventually disappear from crystal form. Consequently, the crystal is bounded by the crystallographic faces having sufficiently low growth rates. From the point of view of statistical thermodynamics, ${ }^{1,2}$ growth kinetics, which depends on the internal structure of the crystals and the external growth conditions, determines the growth rate and therefore the growth form of the crystal. In order to predict the growth morphology, the relation between the growth rate and habitcontrolling factors should be found.

In the past, theories like Bravais-Friedel-Donnay-Harker $(\mathrm{BFDH})$ theory $^{4}$ and Hartman-Perdok theory ${ }^{5-9}$ have been published to predict the growth morphology of crystals. According to BFDH theory, ${ }^{4}$ the relative growth rate of faces $\{h k l\}$ on crystals is taken to be inversely proportional to the interplanar distance $d_{h k l}$,

$$
R_{h k l}^{\text {red }} \sim\left(d_{h k l}\right)^{-1}
$$

$\left(R_{h k l}^{\mathrm{red}}\right.$ denotes the reduced growth rate in the orientations $\{h k l\}$.) Alternatively, in Hartman-Perdok theory ${ }^{5-11}$ the rela- tive growth rate is assumed to be proportional to the magnitude of the attachment energy of faces $\{h k l\}, E_{h k l}^{\text {att }}$, as

$$
R_{h k l}^{\mathrm{red}} \sim E_{h k l}^{\mathrm{att}} \quad \text { or } \quad R_{h k l}^{\mathrm{red}} \sim \eta_{h k l},
$$

with

$$
\begin{gathered}
\eta_{h k l}=E_{h k l}^{\mathrm{att}} / E^{\mathrm{cr}}, \\
E^{\mathrm{cr}}=E_{h k l}^{\mathrm{att}}+E_{h k l}^{\mathrm{slice}} .
\end{gathered}
$$

$\left[E_{h k l}^{\text {att }}\right.$ is the energy released per structural unit when a growth unit is attached to the crystal surface ${ }^{5-11}$ at an appropriate crystallographic position from infinity, $E^{\mathrm{cr}}$ is the lattice energy (per structural unit); $E_{h k l}^{\text {slice }}$ denotes the slice energy, corresponding to the two-dimensional (2D) lattice energy.] These theories are successful to some extent, in the case where crystals are grown from the vapor phase. However, discrepancies between the theoretical and observed morphologies occur frequently, especially when crystals are grown from solutions or the melt.

There are two major problems with these ad hoc recipes. First, according to crystal growth theories, ${ }^{1,2}$ the growth rate of a crystal face is determined by internal and external factors. These two theories take only the internal factors into account. In most cases, the mother phase exerts different influences on different crystal faces. For a given crystal surface, different external conditions may exert very different influences on the growth. This leads to the adoption of crystals with different growth habits. Obviously, the influence of the mother phase is not taken into account in these two theories. Second, the quantitative relation between $R_{h k l}^{\text {red }}$ and habit-controlling parameters has not been properly established yet. Evidently, Eqs. (1) and (2) are too crude to describe the relation between $R_{h k l}^{\text {red }}$ and the habit-controlling 
factors. The aim of this paper is to derive the relation between the relative growth rate $R_{h k l}^{\mathrm{red}}$ and habit-controlling factors based on crystal growth mechanisms. The analysis will be carried out within the framework of the inhomogeneous interfacial cell model. ${ }^{12-14}$ To check the consistency of our theories, the growth morphologies of $n$-paraffin crystals and $\mathrm{C}_{60}$ crystals are examined theoretically and experimentally.

\section{RELATION BETWEEN KINETIC COEFFICIENTS AND INTERFACIAL STRUCTURE}

\section{A. Intermolecular interaction energies at the crystal surface}

The growth of crystals is influenced by the shape of solute molecules, the temperature, concentration, supersaturation, composition of solutions, etc. However, only some of them are face-dependent factors and essentially affect the morphology of crystals. Among those, the effective exchange interaction energies $\phi_{j}$ between solid and fluid units at the interface are one of the most crucial parameters since they influence directly on the kinetics of crystal. ${ }^{1,2}$ (The subscript $j$ denotes the direction of interactions.) In other words, some relevant habit-controlling parameters, like the step energy, the activation energies for the surface integration, etc., are related to $\phi_{j}$.

The expression of $\phi_{j}$ can be obtained on the basis of cell models. ${ }^{13,14}$ According to cell models, ${ }^{1,2,12-14}$ the local enthalpy of dissolution for the crystal surfaces $\{h k l\}, \Delta H_{h k l}^{\text {diss }}$, is expressed as

$$
\Delta H_{h k l}^{\mathrm{diss}}=\sum_{j=1}^{m} \phi_{j},
$$

where $m$ denotes the number of bonds connecting a structural unit with neighboring units. In order to calculate $\phi_{j}$ from $\Delta H_{h k l}^{\text {diss }}$, the proportionality condition should be introduced. ${ }^{9,12,14,15}$ This condition suggests that the interaction energy $\phi_{j}$ is proportional to the corresponding energy between solid-solid units $\Phi_{j}^{\mathrm{SS}}$ or for interactions in different directions:

$$
\phi_{1}: \phi_{2}: \cdots: \phi_{j}: \cdots: \phi_{m}=\Phi_{1}^{\mathrm{SS}}: \Phi_{2}^{\mathrm{SS}}: \cdots: \Phi_{j}^{\mathrm{SS}}: \cdots: \Phi_{m}^{\mathrm{SS}}
$$

From Eq. (6) we find that

$$
\phi_{j} / \sum_{j=1}^{m} \phi_{j}=\Phi_{j}^{\mathrm{SS}} / \sum_{j=1}^{m} \Phi_{j}^{\mathrm{SS}} .
$$

Combining Eq. (7) and Eq. (5) yields

$$
\phi_{j}=\left(\Phi_{j}^{\mathrm{SS}} / 2 E^{\mathrm{cr}}\right) \Delta H_{h k l}^{\mathrm{diss}},
$$

where the lattice energy $E^{\text {cr }}$ is expressed as

$$
E^{\mathrm{cr}}=\frac{1}{2} \sum_{j=1}^{m} \Phi_{j}^{\mathrm{SS}} .
$$

$E^{\mathrm{cr}}$ and $\Phi_{j}^{\mathrm{SS}}$ can be easily calculated from a potential model. ${ }^{1-12}$

Note that we still cannot calculate $\phi_{j}$ based on Eq. (9). Because of the ordering of fluid molecules at the interface and the crystal relaxation near the surface, $\Delta H_{h k l}^{\mathrm{diss}}$ is normally different from the dissolution enthalpy in the bulk phase $\Delta H^{\text {diss }} \cdot{ }^{14,16-25}$ Nevertheless, we may easily estimate $\Delta H_{h k l}^{\text {diss }}$ in terms of a so-called surface scaling factor. ${ }^{14}$ The surface scaling factor $C_{l(h k l)}^{*}$ is defined ${ }^{14}$ as

$$
C_{l(h k l)}^{*}=\Delta H_{h k l}^{\mathrm{diss}} / \Delta H^{\mathrm{diss}} .
$$

Based on regular solution theories, we can verify ${ }^{14,26,27}$ that $C_{l(h k l)}^{*}$ is related to the concentration of the solute in different regions as

$$
C_{l(h k l)}^{*} \cong \ln X_{A(h k l)}^{\mathrm{eff}} / \ln X_{A} .
$$

where $X_{A}$ is the concentration of the solute units in the mother phase and $X_{A(h k l)}^{\text {eff }}$ denotes the concentration of effective growth units at the surface. The so-called effective growth units are defined as follows. Because of ordering and other interfacial effects, fluid units will preferentially adopt a certain orientation and conformation at a given crystal orientation. Those adsorbed solute units which have the orientation and conformation as demanded for the growth in the crystal surface $(h k l)$ can effectively participate in dynamic equilibrium with solid units at the surface. Therefore they are known as effective growth units. In other words, some adsorbed solute units at the crystal surface can be inactive for the crystal growth or in dynamic equilibrium with the crystal. Obviously, for monomer systems, $X_{A(h k l)}=X_{A(h k l)}^{\text {eff }}$ $\left(X_{A(h k l)}\right.$ is the concentration of the adsorbed solute units at the crystal surface). For more details concerning the effective growth units, we refer to Refs. 26, 27.

We notice that $\Delta H^{\text {diss }}$ and $X_{A}$ are the experimentally measurable quantities. Obviously, the key issue here is to calculate $X_{A(h k l)}^{\text {eff }}\left(\right.$ and $\left.X_{A(h k l)}\right)$. Normally, $X_{A(h k l)}^{\text {eff }}\left(\right.$ and $\left.X_{A(h k l)}\right)$ can be calculated using computational techniques, such as molecular-dynamic (MD) computer simulations, Monte Carlo (MC) computer simulations, density-functional-theory calculations, self-consistent-field lattice model calculations, etc. ${ }^{16-21}$ Once $X_{A(h k l)}^{\text {eff }}$ is available, $\phi_{j}$ can be obtained based on Eqs. (8), (10), and (11).

From the point of view of growth morphology, the statistical average of exchange bond energies within the plane parallel to the crystal surface is more important than the exchange bond energy in a specific direction. This energy $\tilde{\phi}_{h k l}$ can also be derived applying the above-mentioned principles. By definition, we have

$$
\tilde{\phi}_{h k l} n_{h k l}=\sum_{j=1}^{n_{h k l}} \phi_{j}
$$

where $n_{h k l}$ is the coordination number of a structural unit within the two-dimensional (2D) crystal slice $(h k l)$.

Similar to $E^{\text {cr }}$, the slice energy $E_{h k l}^{\text {slice }}$ can be expressed as

$$
E_{h k l}^{\text {slice }}=\frac{1}{2} \sum_{j=1}^{n_{h k l}} \Phi_{j}^{\mathrm{SS}} .
$$

Based on the proportionality condition, we may reduce $\Delta H_{h k l}^{\text {diss }}$ to a sum of in-plane contributions by multiplying it by $E_{h k l}^{\text {slice }} / E^{\mathrm{cr}}=\left(1-\eta_{h k l}\right)$ [cf. Eqs. (3), (4), (9), and (13)]. According to Eqs. (10) and (12), we approximate 


$$
\tilde{\phi}_{h k l}=\sum_{j=1}^{n_{h k l}} \phi_{j} / n_{h k l} \approx\left(1-\eta_{h k l}\right) C_{l(h k l)}^{*} \Delta H^{\mathrm{diss}} / n_{h k l} .
$$

\section{B. Step energy and kinetic coefficients}

The growth of crystals is actually a process to deliver growth units from the bulk phase to kink sites at the crystal surface and incorporate them into the crystal. ${ }^{28,29}$ This process can be roughly summarized in the following ways:

$$
\begin{aligned}
& \text { (i) growth units } \longrightarrow \text { bulk diffusion } \\
& \text { (in the bulk) } \\
& \text { adsorbed units } \\
& \text { (at the crystal surface) }
\end{aligned}
$$

Note that, generally speaking, the average distance between two kinks at a step $\lambda_{0}$ is a few atomic dimensions. Almost all growth units adsorbed at steps can easily reach kinks via one-dimensional diffusion. Therefore, during crystal growth, we believe that all growth units which reach steps can also reach kinks.

For the growth of a crystal face, the kinetics depends strongly on the step energy $\phi_{j}^{\text {step }}$ (and the step free energy $\gamma_{j}$ ). Some kinetic parameters, such as $\lambda_{s}, D_{s} \beta_{\text {st }}$, etc., also play a very important role. In order to derive the relative growth rate in different crystallographic orientations, we first need to estimate $\phi_{j}^{\text {step }}$ (and $\gamma_{j}$ ) and the kinetic parameters at the crystal surface. $\lambda_{s}$ is the diffusion mean free path, $D_{s}$ is the surface diffusion constant, and $\beta_{\text {st }}$ is the kinetic coefficient for the step integration. The last three factors can be expressed $^{28,29}$ as

$$
\begin{gathered}
\beta_{\mathrm{st}} \approx a \nu_{\|}\left(a / \lambda_{0}\right) \exp \left(-\Delta G_{\mathrm{step}}^{\neq} / k_{b} T\right) \\
\lambda_{s} \approx a\left(\nu_{\|} / \nu_{\perp}\right)^{1 / 2} \exp \left[\left(\Delta G_{\text {deads }}^{\neq}-\Delta G_{\text {sdiff }}^{\neq}\right) / 2 k_{b} T\right] \\
\approx a \exp \left[\left(\Delta G_{\text {deads }}^{\neq}-\Delta G_{\text {sdiff }}^{\neq}\right) / 2 k_{b} T\right] \quad\left(\nu_{\|} \approx \nu_{\perp}\right), \\
D_{s} \approx \frac{1}{4}\left(a^{2} \nu_{\|}\right) \exp \left(-\Delta G_{\text {sdiff }}^{\neq} / k_{b} T\right)
\end{gathered}
$$

$\left(\Delta G_{\text {step }}^{\neq}\right.$is the activation energy for a growth unit to enter a kink, $\Delta G_{\text {deads }}^{\neq}$is the potential barrier of deadsorption of an adatom, $\Delta G_{\text {sdiff }}^{\neq}$is the potential barrier of the surface diffusion, $\lambda_{0}$ is the average distance between the kinks, and $\nu_{\|}$and $\nu_{\perp}$ are the frequency of thermal vibration parallel and normal to the surface for adatoms, respectively.) For the growth from vapor or solution, different states of a growth unit at the surface and the activation energies that must be overcome to enter a kink are illustrated Fig. 1.

Here the average step (free) energy $\phi_{h k l}^{\text {step }}$ is approximately equal to $\tilde{\phi}_{h k l} \cdot{ }^{12,14}$ It then follows from Eq. (14) that the average step energy of the faces $\{h k l\}$ is given by

$$
\tilde{\phi}_{h k l}^{\text {step }} / k_{b} T \approx\left(1-\eta_{h k l}\right) C_{l(h k l)}^{*} \varepsilon / n_{h k l},
$$

and in the case where the temperature $T$ is below and not very close to the roughening temperature $T^{R}$,

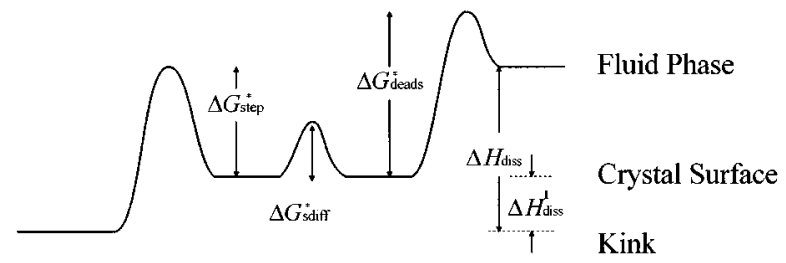

FIG. 1. Schematized states of a growth unit and potential barriers that must be overcome in order that the growth unit can enter a kink.

$$
\gamma / k_{b} T \approx \tilde{\phi}_{h k l}^{\mathrm{step}} / k_{b} T \approx C_{l(h k l)}^{*}\left(1-\eta_{h k l}\right) \varepsilon / n_{h k l} .
$$

Here $\varepsilon=\Delta H / k_{b} T$ ( $\Delta H$ denotes the molar enthalpy of dissolution, melting, or evaporation).

Looking at Eq. (15), we note that the kink density $a / \lambda_{0}$ can be directly related to the step energy $\tilde{\phi}_{h k l}^{\mathrm{kink}} / k_{b} T .^{27,28}$ Since $\tilde{\phi}_{h k l}^{\text {kink }} \approx \tilde{\phi}_{h k l}^{\text {step }},{ }^{27}$ it is given, in the case $a \ll \lambda_{0}$, by

$$
a / \lambda_{0} \approx 2 \exp \left(-\tilde{\phi}_{h k l}^{\mathrm{step}} / k_{b} T\right) .
$$

Therefore, to estimate $\beta_{\text {st }}$ is a question of the estimation of the activation energy $\Delta G_{\text {step }}^{\neq}$. Similarly, for $\lambda_{s}$ and $D_{s}$, the estimation of $\Delta G_{\text {deads }}^{\neq}$and $\Delta G_{\text {sdiff }}^{\neq}$is the crucial point. We notice that the exact calculation of these activation energies is very difficult or impossible in most cases. Therefore it is necessary to estimate their values by introducing certain approximations.

Let us first focus our attention on $\Delta G_{\text {deads }}^{\neq}$and $\Delta G_{\text {sdiff }}^{\neq}$. In simple cases, the deadsorption activation energy of a molecule from the surface is approximately equal to the difference in energy between the two different states. This implies that the deadsorption of an admolecule from the crystal surface is associated with breaking the vertical solid-fluid bonds at the surface. ${ }^{8,28,29}$ Therefore we take $\Delta G_{\text {deads }}^{\neq}$proportional to the magnitude of the local attachment energy at the surface $\left[(1 / 2) \eta_{h k l} C_{l(h k l)}^{*} \Delta H\right]$. For the surface diffusion, the potential barrier $\Delta G_{\text {sdiff }}^{\neq}$is supposed to be a small fraction $(t)$ of $\Delta G_{\text {deads }}^{\neq}$. (We assume that normally $t \sim 1 / 4$.) It then follows that

$$
\Delta G_{\mathrm{deads}}^{\neq} / k_{b} T \approx \eta_{h k l} C_{l(h k l)}^{*} \varepsilon / 2
$$

and

$$
\Delta G_{\mathrm{sdiff}}^{\neq} / k_{b} T \approx t C_{l(h k l)}^{*} \eta_{h k l} \varepsilon / 2+\Delta G^{\prime} / k_{b} T .
$$

Here $\Delta G^{\prime}$ represents the activation free energy for the resistance against movement due to adsorbed fluid units.

The activation energy $\Delta G_{\text {step }}^{\neq}$is a very complicated factor. This activation energy depends on the configuration of the activated complex in the liquid, i.e., on the disposition of the nearest neighbors of the particle shifting from the liquid to the solid phase. It includes the potential barriers of desolvation, rearrangement of the short-range order at the steps, etc. These potential barriers are associated with the ordering of fluid units and the intermolecular structure at the solid-fluid interface. During the growth of chemically simple substances (elements or substances with high-symmetry molecules) from the gas phase without chemical reactions, $\Delta G_{\text {step }}^{\neq}$is close to zero. ${ }^{28}$ In the case of complex molecules crystallizing from the melt or solutions, the above-mentioned effects 
are very pronounced. Therefore an appreciate effective barrier must exist for them. Henceforth, we approximate here

$$
\Delta G_{\mathrm{step}}^{\neq} / k_{b} T=t_{h k l}+\Delta G^{\prime \prime} / k_{b} T
$$

with

$$
t_{h k l} \approx-\ln \left[X_{A(h k l)}^{\mathrm{eff}} / X_{A(h k l)}\right]
$$

[See the Appendix for Eq. (24).] Here $t_{h k l}$, called the molecular orientational factor, is used to characterize the potential barrier for asymmetric growth units to orient themselves in order to be incorporated into the steps. $\Delta G^{\prime \prime}$ corresponds to the desolvation activation energy for an adsorbed growth unit to enter the kink site.

It follows from (19)-(24) that

$$
\begin{gathered}
\lambda_{s} \approx a \exp \left[(1-t) C_{l(h k l)}^{*} \eta_{h k l} \varepsilon / 4\right], \\
D_{s}=\left(a^{2} \nu_{\|}\right) / 4 \exp \left(-\frac{1}{2} t C_{l(h k l)}^{*} \eta_{h k l} \varepsilon-\Delta G^{\prime} / k_{b} T\right),
\end{gathered}
$$

and

$$
\beta_{\mathrm{st}} \approx 2 a \nu_{\|} \exp \left[-\left(1-\eta_{h k l}\right) C_{l(h k l)}^{*} \varepsilon / n_{h k l}-t_{h k l}-\Delta G^{\prime \prime} / k_{b} T\right]
$$

Based on the above analyses, we make two reasonable assumptions: (1) $\Delta G^{\prime}$ is roughly equal to $\Delta G^{\prime \prime}$. (2) $\Delta G^{\prime} / 2 k_{b} T$ is so small compared to $(1 / 4)(1-t) C_{l(h k l)}^{*} \eta_{h k l} \varepsilon$ that it can be neglected. These two assumptions are very relevant for the derivations in the following sections.

\section{RELATIVE GROWTH RATE AND HABIT-CONTROLLING FACTORS}

\section{A. Screw dislocation mechanism}

In this part, we will follow the presentation and discussion of the screw dislocation mechanism by Chernov. ${ }^{28}$

Within the framework of this mechanism, ${ }^{1,2,28,29}$ a growing crystal is supposed to be imperfect, due to the presence of screw dislocations. Screw dislocations provide the crystal surfaces with permanent step sources, which allows the crystals to grow continuously. When the growth of a crystal face is governed by a screw dislocation, it is reasonable to assume that the step integration is a rate determining step in the whole growth process. Then the normal growth rate of the crystal surface is given by

$$
R_{h k l}=h_{s} k_{b} T \sigma \hat{V} /(19 \gamma a)
$$

with $h_{s}=$ step height (in the following, we assume that the steps occurring at the surface are monosteps: then, $h_{s}=d_{h k l}$ ), $a=$ dimension of a structural unit parallel to the surface, $\gamma=$ step free energy per length unit, the supersaturation $\sigma=\Delta \mu / k_{b} T(\Delta \mu$ is the difference in chemical potential between solute and solid units), and $\hat{V}=$ velocity of a step. The expression of $\hat{V}$ depends on the way growth units are transported from the bulk to the steps on the surface. As crystals are grown from different mediums, the major way of delivery of the crystallizing substance to the kinks will be either bulk or surface diffusion.

\section{Surface diffusion model}

For crystals growing from vapor or sometimes from solution, surface diffusion plays a leading role in the delivery of the crystallizing substance to the steps [cf. case (i) in Sec. II B]. It follows from the Burton-Cabrera-Frank (BCF) model $^{28}$ that the step velocity has the form

$$
\hat{V}=\sigma \lambda_{s} \nu_{\perp} \zeta \exp \left(-\Delta H / k_{b} T\right) \tanh \left(\sigma_{1} / \sigma\right),
$$

with the retardation factor

$$
\zeta=\left[1+D_{s} /\left(\beta_{\mathrm{st}} \lambda_{s}\right) \tanh \left(\sigma_{1} / \sigma\right)\right]^{-1}
$$

and the factor

$$
\sigma_{1}=19 \gamma a /\left(k_{b} T \lambda_{s}\right)
$$

From the discussions in last section, we then arrive at the expressions

$$
\sigma_{1} \approx 19\left(1-\eta_{h k l}\right) C_{l(h k l)}^{*} \varepsilon \exp \left[-(1-t) C_{l(h k l)}^{*} \eta_{h k l} \varepsilon / 4\right] / n_{h k l}
$$

and

$$
\begin{aligned}
\zeta \approx & \left\{1+\exp \left[-\frac{1}{4}(1+t) C_{l(h k l)}^{*} \eta_{h k l} \varepsilon+t_{h k l}\right.\right. \\
& \left.\left.+\left(1-\eta_{h k l}\right) C_{l(h k l)}^{*} \varepsilon / n_{h k l}\right] / 8 \tanh \left(\sigma_{1} / \sigma\right)\right]^{-1} .
\end{aligned}
$$

With (29), (32), and (33), expression (28) becomes

$$
\begin{aligned}
R_{h k l} \approx & n_{h k l} d_{h k l} \zeta \exp (-\varepsilon)(\sigma)^{2}\left[19\left(1-\eta_{h k l}\right) C_{l(h k l)}^{*} \varepsilon\right]^{-1} \\
& \times \exp \left[(1-t) C_{l(h k l)}^{*} \eta_{h k l} \varepsilon / 4\right] \tanh \left(\sigma_{1} / \sigma\right)
\end{aligned}
$$

Leaving out of consideration the orientation-independent factors, the reduced growth rate $R_{h k l}^{\text {red }}$ of the face $(h k l)$ obtains the form

$$
\begin{aligned}
R_{h k l}^{\mathrm{red}} \sim & n_{h k l} d_{h k l} \zeta\left[\left(1-\eta_{h k l}\right) C_{l(h k l)}^{*}\right]^{-1} \\
& \times \exp \left[(1-t) \eta_{h k l} C_{l(h k l)}^{*} \varepsilon / 4\right] \tanh \left(\sigma_{1} / \sigma\right)
\end{aligned}
$$

It can be seen from Eq. (33) that the retardation factor $\zeta$ is also an important habit-controlling factor. This factor also depends on the wetting condition (characterized by $C_{l(h k l)}^{*}$ ), the orientation factor $\eta_{h k l}$, the specific enthalpy of dissolution $\varepsilon$, and the supersaturation $\sigma$. According to (33), $\zeta$ increases with $\eta_{h k l}$ or decreases with $C_{l(h k l)}^{*}$. In addition, $\zeta$ is also influenced by $\sigma$ ( $\zeta$ will increase with $\sigma$ ). In Fig. 2(a), $\zeta$ is plotted versus $\eta_{h k l}$ and $\sigma$ for a system with $\varepsilon=8, n_{h k l}=4$, and $C_{l(h k l)}^{*}=0.5$. $\left[t_{h k l}\right.$ can be to some extent related to $C_{l(h k l)}^{*}$. For the sake of illustration and simplicity, we assume that $t_{h k l} \sim\left(1-\eta_{h k l}\right) C_{l(h k l)}^{*} \varepsilon / n_{h k l}$ hereafter. In practice, $t_{h k l}$ should be calculated according to Eq. (24).] Also for the same system, $\zeta$ is plotted versus $C_{l(h k l)}^{*}$ and $\sigma$ for $\eta_{h k l}=0.5$ [see Fig. 2(b)]. It can be seen from these plots that $\zeta$ increases with $\eta_{h k l}$ or decreases with $C_{l(h k l)}^{*}$. In addition, $\zeta$ is also influenced by $\sigma$ ( $\zeta$ will increase with $\sigma$ ). However, it can be found from Fig. 2 that if $C_{l(h k l)}^{*}$ is not very low and $\eta_{h k l}$ is not too high, $\zeta$ is approximately independent of $\sigma$ $\left[\tanh \left(\sigma_{1} / \sigma\right) \approx 1\right.$ in the case $\left.\sigma<\sigma_{1}\right]$. This suggests that if $\sigma$ is relatively low, $\zeta$ is only a function of $C_{l(h k l)}^{*}, \eta_{h k l}$, and $\varepsilon$. In this case, Eq. (33) can be rewritten as 

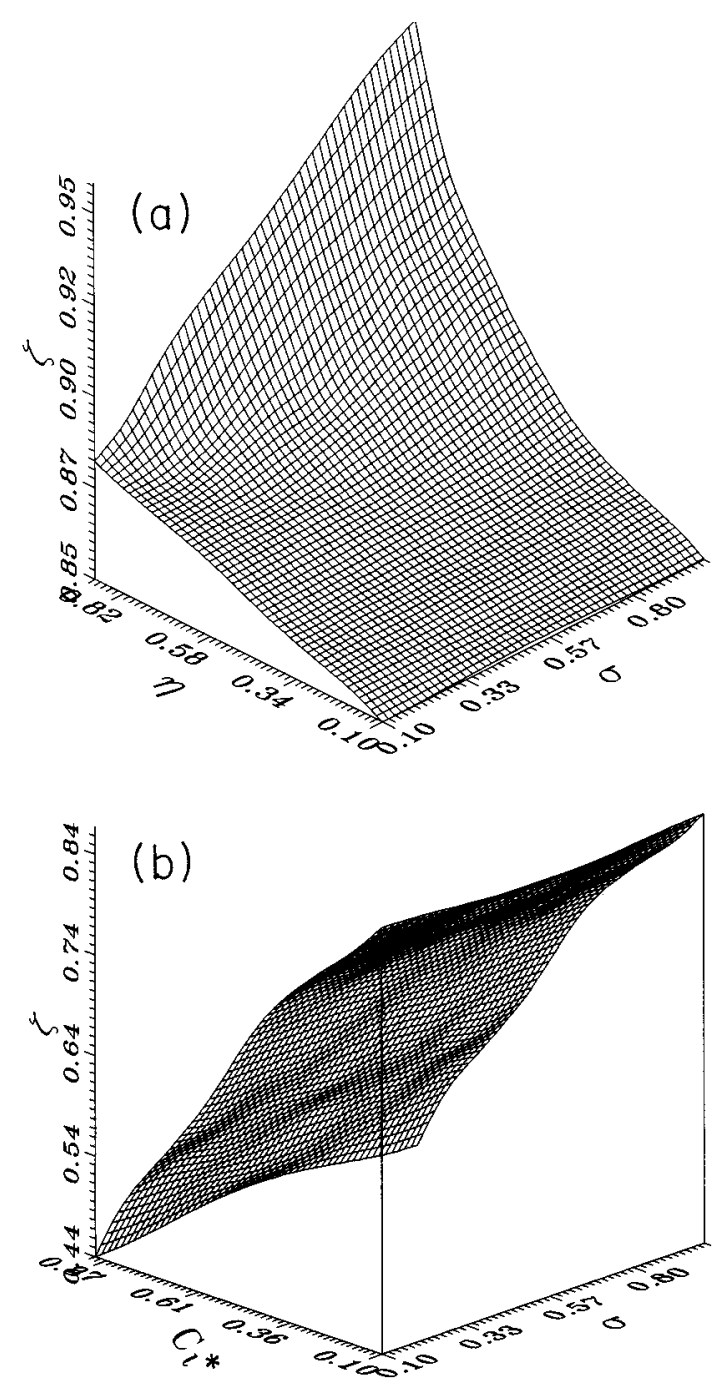

FIG. 2. Retardation factor $\zeta$ as a function of $\eta_{h k l}$ (a) and of $C_{l(h k l)}^{*}$ (b) for different supersaturations $\sigma . \quad \varepsilon=8, n_{h k l}=4$, and $t \approx 0.25$; (a) $C_{l(h k l)}^{*}=0.1$ and (b) $\eta_{h k l}=0.4$ [Eq. (33)].

$$
\begin{aligned}
\zeta \approx & \left\{1+\exp \left[-(1+t) C_{l(h k l)}^{*} \eta_{h k l} \varepsilon / 4+t_{h k l}\right.\right. \\
& \left.\left.+\left(1-\eta_{h k l}\right) C_{l(h k l)}^{*} \varepsilon / n_{h k l}\right] / 8\right\}^{-1} .
\end{aligned}
$$

Similarly to $\zeta$, the dependent relation between $R_{h k l}^{\text {red }}$ and habit-controlling factors can be analyzed based on Eq. (35). Figure 3(a) shows (35) the relation between $R_{h k l}^{\text {red }} / d_{h k l}$ and $\eta_{h k l}$ at different supersaturations $\sigma\left(n_{h k l}=4, \varepsilon=6, C_{l(h k l)}^{*}\right.$ $=0.5$, and $t=1 / 4) . \quad R_{h k l}^{\mathrm{red}} / d_{h k l}$ increases drastically with $\eta_{h k l}$ at low supersaturations, while it only increases smoothly at high supersaturations. Within the framework of this mechanism, crystals will acquire more anisotropic forms at relatively low supersaturations than at high supersaturations. It is interesting to note that at high supersaturations $R_{h k l}^{\text {red }}$ $\sim d_{h k l} \eta_{h k l}$. This implies that crystals acquire a morphology similar to the equilibrium morphology $\left[D_{h k l} \sim d_{h k l} \eta_{h k l} ; D_{h k l}\right.$ is the distance from the center to the faces $\{h k l\}$ (Refs. 1, 2)].

Figure 3(b) shows the dependence of $R_{h k l}^{\mathrm{red}} / d_{h k l}$ on $C_{l(h k l)}^{*}$ at different supersaturations. In contrast to $\eta_{h k l}$, the reduced growth rate decreases with $C_{l(h k l)}^{*}$. This can be explained from the point of view of crystal growth. It follows from the discussions in Sec. II that for a given solid-fluid interface, lower values of $C_{l(h k l)}^{*}$ correspond to the lower step (free) energies. This implies that the potential barrier for the growth is lower. Therefore the growth rate will be higher. The change in the growth rate due to the change of $C_{l(h k l)}^{*}$ becomes less pronounced at high supersaturation. This tendency is similar to $\eta_{h k l}$.

In addition to $\eta_{h k l}, C_{l(h k l)}^{*}$, and $\sigma, \varepsilon$ also influence $R_{h k l}^{\text {red }}$. In Figs. 3(c), 3(d), $R_{h k l}^{\mathrm{red}} / d_{h k l}$ is plotted versus $\eta_{h k l}\left(n_{h k l}=4\right.$, $C_{l(h k l)}^{*}=0.5, t=0.25, t_{h k l}=1$, and $\left.\sigma=0.1\right)$, and $R_{h k l}^{\text {red }} / d_{h k l}$ is plotted versus $C_{l(h k l)}^{*}\left(n_{h k l}=4, \quad \eta_{h k l}=0.4, \quad \sigma=0.1\right.$, and $t=0.25)$ for various values of $\varepsilon$. We can see from these two figures that the shape of the $R_{h k l}^{\text {red }}$ curves does not change much by $\varepsilon$. However, the magnitude of the change in $R_{h k l}^{\text {red }} / d_{h k l}$ with respect to $\eta_{h k l}$ or $C_{l(h k l)}^{*}$ is altered by $\varepsilon$. In this sense, $\varepsilon$ can be regarded as "an amplifier" for the influence of other habit-controlling factors.

We notice that Eq. (35) is a general expression derived from the BCF model. Under certain conditions, it can be further simplified. In the following, three cases are distinguished.

(i) The case where the supersaturation is relatively low ( $\sigma$ $\left.<\sigma_{1}\right)$. In this case, one has $\tanh \left(\sigma_{1}<\sigma\right) \approx 1$. Similarly to $\zeta$ given by Eq. (33), $R_{h k l}^{\text {red }}$ can also be expressed independently of $\sigma$ as

$$
\begin{aligned}
R_{h k l}^{\mathrm{red}} \sim & n_{h k l} d_{h k l} \zeta\left[\left(1-\eta_{h k l}\right) C_{l(h k l)}^{*}\right]^{-1} \\
& \times \exp \left[(1-t) \eta_{h k l} C_{l(h k l)}^{*} \varepsilon / 4\right] .
\end{aligned}
$$

This implies that $R_{h k l}^{\text {red }}$ does not depend on the supersaturation. Notice that since $\sigma_{1}$ is also a function of $\eta_{h k l}$, $C_{l(h k l)}^{*}$, and $\varepsilon$, the crystal orientations under consideration should exhibit very large differences in $\eta_{h k l}$ and $C_{l(h k l)}^{*}$.

(ii) The case $D_{s} /\left(\beta_{\mathrm{st}} \lambda_{s}\right) \ll 1$ or $\zeta \sim 1$. This happens when $\eta_{h k l}$ is large or $C_{l(h k l)}^{*}$ is rather small and $\sigma$ is relatively large. One obtains from (35) the expression

$$
\begin{aligned}
R_{h k l}^{\mathrm{red}} \sim & n_{h k l} d_{h k l}\left[\left(1-\eta_{h k l}\right) C_{l(h k l)}^{*}\right]^{-1} \\
& \times \exp \left[(1-t) \eta_{h k l} C_{l(h k l)}^{*} \varepsilon / 4\right] \tanh \left(\sigma_{1} / \sigma\right)
\end{aligned}
$$

(iii) The case $D_{s} /\left(\beta_{\mathrm{st}} \lambda_{s}\right) \gg 1$, or $\zeta \ll 1$. Here the so-called second parabolic law ${ }^{29}$ is applied. This happens when the growth units enter the solution directly from the kinks of the steps. Equation (35) can be rewritten as

$$
\begin{aligned}
R_{h k l}^{\mathrm{red}} \sim & n_{h k l} d_{h k l}\left[\left(1-\eta_{h k l}\right) C_{l(h k l)}^{*}\right]^{-1} \\
& \times \exp \left\{\left[\eta_{h k l}-\left(1-\eta_{h k l}\right) / n_{h k l}\right] C_{l(h k l)}^{*} \varepsilon / 2-t_{h k l}\right\}
\end{aligned}
$$

It can be seen from (38) that $R_{h k l}^{\mathrm{red}}$ is also independent of $\sigma$ for the entire range of supersaturation.

\section{Chernov's model}

In the case that crystals are grown from solutions, it is assumed that the growth units are mainly transported to the 

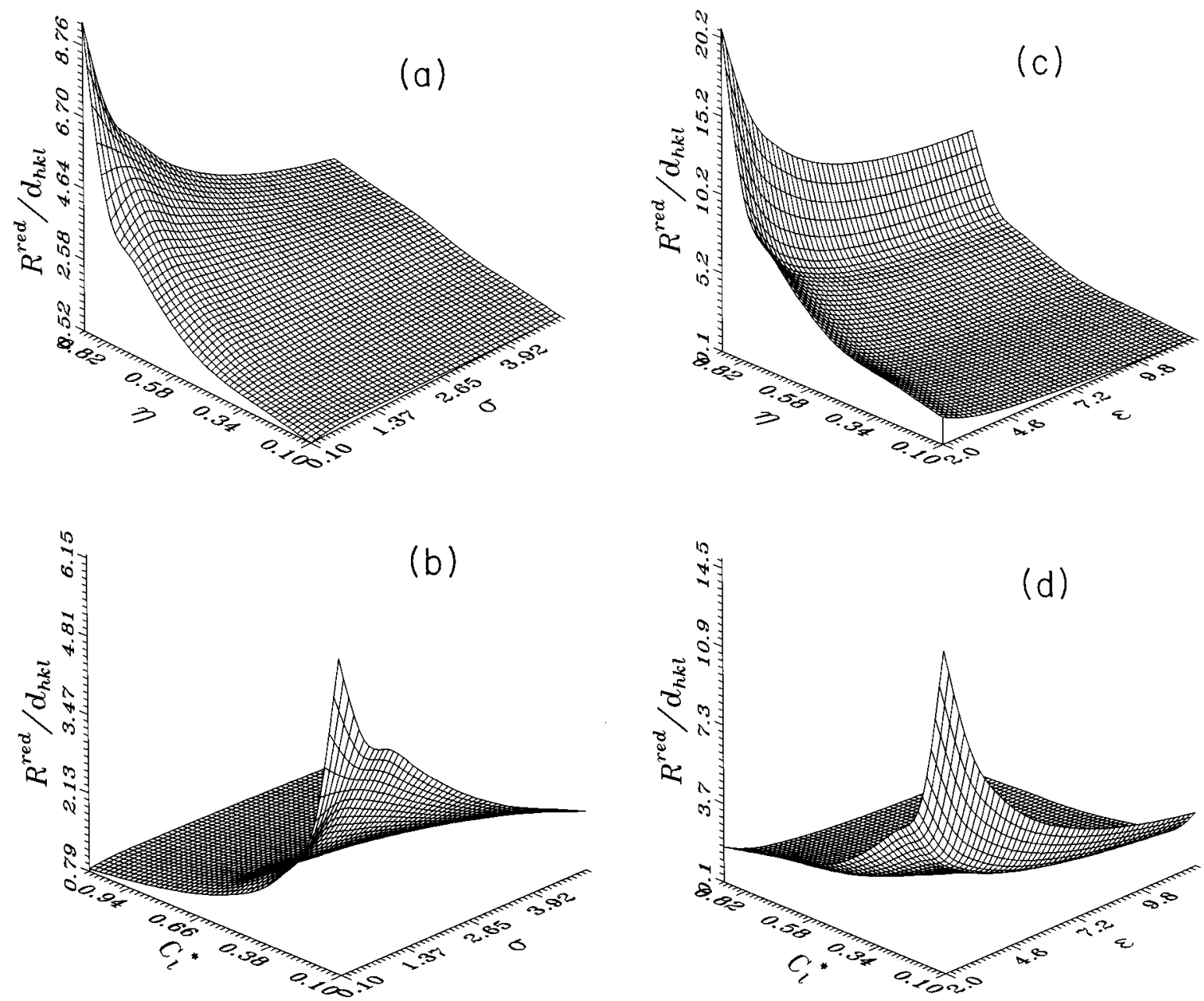

FIG. 3. Reduced growth rate as a function of various parameters [Eq. (35)] the BCF model. (a) $R_{h k l}^{\text {red }} / d_{h k l}$ plotted vs $\eta_{h k l}$ for various supersaturations $\sigma ; \varepsilon=6, n_{h k l}=4, C_{l(h k l)}^{*}=0.5, t=0.25$. (b) $R_{h k l}^{\text {red }} / d_{h k l}$ plotted vs $C_{l(h k l)}^{*}$ for various supersaturations $\sigma ; n_{h k l}=2, \varepsilon=6$, $\eta_{h k l}=0.5, t=0.25, t_{h k l}=1$. (c) $R_{h k l}^{\mathrm{red}} / d_{h k l}$ plotted vs $\eta_{h k l}$ for various $\varepsilon ; n_{h k l}=4, C_{l(h k l)}^{*}=0.5, t=0.25, \sigma=0.1$. (d) $R_{h k l}^{\mathrm{red}} / d_{h k l} \mathrm{plotted} \mathrm{vs}$ $C_{l(h k l)}^{*}$ for various $\varepsilon ; n_{h k l}=4, \eta_{h k l}=0.4, \sigma=0.1, t=0.25$.

kinks of the steps by means of volume diffusion [cf. case (ii) in Sec. II B]. According to Chernov, ${ }^{28}$ the step rate is given by

$$
\hat{V}=\beta_{\mathrm{st}} \zeta^{\prime} \Omega X_{A(h k l)}^{\mathrm{eq}} \sigma,
$$

with

$$
\zeta^{\prime}=\left[1+\beta_{\mathrm{st}} d_{h k l}(\pi D)^{-1} \ln \left(\lambda / d_{h k l}\right) \sinh \left(\pi \delta^{\neq} / \lambda\right)\right]^{-1} .
$$

Here $\delta^{\neq}$is the thickness of the diffusion boundary layer, $D$ is the diffusivity, $\lambda$ is the distance between two parallel steps, and $X_{A(h k l)}^{\mathrm{eq}}$ is the equilibrium concentration of solute molecules at the crystal surface.

Following from Sec. II B, $\lambda \approx 19 \gamma a /\left(k_{b} T \sigma\right) \approx 19(1$ $\left.-\eta_{h k l}\right) C_{l(h k l)}^{*} \varepsilon a /\left(n_{h k l} \sigma\right)$. Therefore one obtains

$$
\begin{aligned}
\zeta^{\prime}= & \left\{1+\beta_{\mathrm{st}} d_{h k l}(\pi D)^{-1} \ln \left[\sigma_{2} \pi \delta^{\neq} /\left(\sigma d_{h k l}\right)\right]\right. \\
& \left.\times \sinh \left(\sigma / \sigma_{2}\right)\right\}^{-1},
\end{aligned}
$$

with

$$
\sigma_{2} \approx 19\left(1-\eta_{h k l}\right) C_{l(h k l)}^{*} \varepsilon a /\left(n_{h k l} \pi \delta^{\neq}\right) .
$$

Substituting Eqs. (39)-(42) into (28) yields

$$
\begin{aligned}
R_{h k l} \approx & n_{h k l} d_{h k l} k_{b} T \zeta^{\prime} \beta_{\mathrm{st}} \Omega X_{A(h k l)}^{\mathrm{eq}} \\
& \times\left[19\left(1-\eta_{h k l}\right) C_{l(h k l)}^{*} \varepsilon a\right]^{-1} \sigma^{2}
\end{aligned}
$$

Note that $X_{A(h k l)}^{\mathrm{eq}}$, according to Eqs. (11) and (24), is equal to

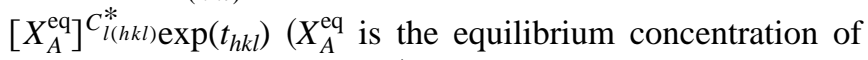
solute molecules in the bulk). In case that the supersaturation is relatively low, $X_{A}^{\text {eq }}$ can be approximately expressed by $X_{A}$. Otherwise, the 't Hoff equation should be applied to calculated $X_{A}^{\mathrm{eq}}$.

In analogy with Eq. (36), two cases can be also distinguished.

Case I, $\pi \delta^{\ddagger} \ll \lambda$. This implies that $\sigma \ll \sigma_{2}$. It then follows that $\sinh (\pi \delta / \lambda) \approx \pi \delta^{ \pm} / \lambda^{28}$ In this case, we have $\beta_{\mathrm{st}} d_{h k l} \delta^{\neq}(D \lambda)^{-1} \ll 1$ (or $\left.\zeta^{\prime} \sim 1\right){ }^{28}$ Therefore Eq. (43) is rewritten as 


$$
R_{h k l} \approx 2 n_{h k l} d_{h k l} \nu_{\|} \Omega\left[X_{A}\right]^{C_{l(h k l)}^{*}} \sigma^{2}\left[19\left(1-\eta_{h k l}\right) C_{l(h k l)}^{*} \varepsilon\right]^{-1} \exp \left[-\left(1-\eta_{h k l}\right) C_{l(h k l)}^{*} \varepsilon / n_{h k l}-\Delta G^{\prime \prime} / k_{b} T\right] .
$$

Applying the 't Hoff equation for $X_{A}^{\mathrm{eq}}$, Eq. (44) can be rewritten as

$$
R_{h k l} \approx 2 n_{h k l} d_{h k l} \nu_{\|} \Omega \sigma^{2}\left[19\left(1-\eta_{h k l}\right) C_{l(h k l)}^{*} \varepsilon\right]^{-1} \exp \left[-\left(1-\eta_{h k l}\right) C_{l(h k l)}^{*} \varepsilon / n_{h k l}-\left(T^{m}-T\right) T^{m} C_{l(h k l)}^{*} \varepsilon-\Delta G^{\prime \prime} / k_{b} T\right] .
$$

According to Hartman and Bennema, ${ }^{8}$ the desolvation activation energies $\Delta G^{\prime \prime}$ is supposed to be face independent. This can only be true when the interactions between adsorbed solvent or impurity molecules and solid molecules are weak and $t_{h k l}$ is relatively low. It then follows that

$$
R_{h k l}^{\mathrm{red}} \sim n_{h k l} d_{h k l}\left[X_{A}\right]_{l(h k l)}^{C_{l}^{*}}\left[\left(1-\eta_{h k l}\right) C_{l(h k l)}^{*}\right]^{-1} \exp \left[-\left(1-\eta_{h k l}\right) C_{l(h k l)}^{*} \varepsilon / n_{h k l}\right]
$$

or

$$
R_{h k l}^{\mathrm{red}} \sim n_{h k l} d_{h k l}\left[\left(1-\eta_{h k l}\right) C_{l(h k l)}^{*}\right]^{-1} \exp \left[-\left(1-\eta_{h k l}\right) C_{l(h k l)}^{*} \varepsilon / n_{h k l}-\left(T^{m}-T\right) T^{m} C_{l(h k l)}^{*} \varepsilon\right] .
$$

In the case where solvent and impurity molecules are quite similar to solute molecules and have strong interactions with crystal faces or solute molecules are highly asymmetrical, the desolvation activation energy $\Delta G^{\prime \prime}$ shows a strong facedependent character. This implies that from Eqs. (44) and (45) [or from Eqs. (44a) and (45a)] the term $\Delta G^{\prime \prime} / k_{b} T$ cannot be left out of consideration. Thus, Eqs. (45), (45a) should be rewritten as

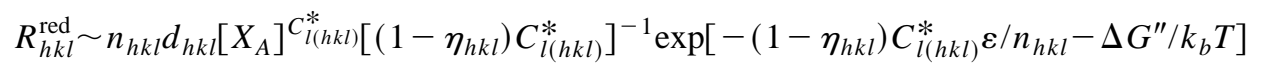

or

$$
R_{h k l}^{\mathrm{red}} \sim n_{h k l} d_{h k l}\left[\left(1-\eta_{h k l}\right) C_{l(h k l)}^{*}\right]^{-1} \exp \left[-\left(1-\eta_{h k l}\right) C_{l(h k l)}^{*} \varepsilon / n_{h k l}-\left(T^{m}-T\right) T^{m} C_{l(h k l)}^{*} \varepsilon-\Delta G^{\prime \prime} / k_{b} T\right]
$$

To show the dependence of $R_{h k l}^{\text {red }}$ on various factors, $R_{h k l}^{\mathrm{red}} / d_{h k l}$ is plotted as a function of $\eta_{h k l}$ and $C_{l(h k l)}^{*}\left(n_{h k l}=4\right.$, $\varepsilon=6, X_{A} \sim 1$ ) based on Eq. (45) (see Fig. 4). It can be seen that $R_{h k l}^{\text {red }}$ depends very strongly on $C_{l(h k l)}^{*}$ and $\eta_{h k l}$. The morphology of crystals depends also on the concentration of the solute or the crystallization temperature. Referring to Eqs. (45), (45a), (46), and (46a), it can be visualized that with decreasing the concentration or the crystallization temperature, crystals will obtain more anisotropic growth forms. In spite of this, $R_{h k l}^{\text {red }}$ is also influenced by $\varepsilon$ in the similar way as in the surface diffusion model.

Case II, $\pi \delta^{\neq} \gg \lambda$ or $\sigma \gg \sigma^{2}$. This implies that

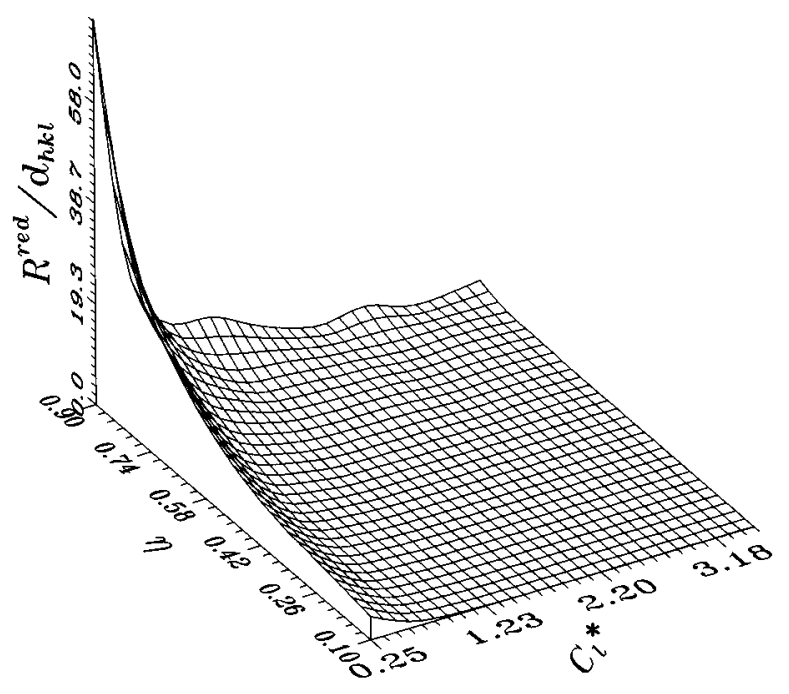

FIG. 4. Reduced growth rate $R_{h k l}^{\text {red }} / d_{h k l}$ plotted as a function of $\eta_{h k l}$ and $C_{l(h k l)}^{*}, n_{h k l}=4, \varepsilon=6$. The Chernov model [Eq. (46)]. $\beta_{\mathrm{st}} d_{h k l} \delta^{\neq}(D \lambda)^{-1} \gg 1$. In this case, the growth rate is mainly determined by orientation-independent factors, ${ }^{28}$

$$
R_{h k l} \approx \Omega\left[X_{A}^{\mathrm{eq}}\right]^{C_{l(h k l)}^{*} D \sigma / \delta^{\neq}}
$$

Therefore,

$$
R_{h k l}^{\mathrm{red}} \sim\left[X_{A}^{\mathrm{eq}}\right]^{C_{l(h k l)}^{*},}
$$

implying that all faces have the same growth rate at $X_{A}^{\mathrm{eq}} \sim 1$ and crystals may obtain the shape of a sphere. In practice, however, as has been shown by Bennema et al. ${ }^{1,2}$ this limit is never reached. On the other hand, if $X_{A}^{\mathrm{eq}}<1$, crystals will obtain anisotropic growth form due to $C_{l(h k l)}^{*}$. As mentioned before, the degree of anisotropy of crystals will increase with decreasing $X_{A}^{\mathrm{eq}}$.

\section{B. Two-dimensional nucleation mechanism}

This mechanism has been discussed by many authors. Here we adopt the mechanism discussed by Lewis, ${ }^{30}$ according to which the following two cases occur.

\section{Mononuclear model}

In this model one nucleus grows on a crystal face and is covering the surface entirely before a second nucleus appears. Then 


$$
R_{h k l}=B \exp \left(-\Delta G^{*} / k_{b} T\right) .
$$

Here $\Delta G^{*}$ is the Gibbs free energy (per growth unit) for the formation of a critical two-dimensional nucleus and $B$ is the kinetic coefficient depending on the growth conditions and crystallographic orientation:

$$
B=L^{2} K \sigma C^{\prime} \lambda_{s}^{2},
$$

where $L$ is the edge length of a crystal face, $C^{\prime}=\zeta C_{e}$, and $C_{e}$ is the equilibrium concentration in vapor or liquid, while $\zeta$ is the retardation factor of the BCF theory [see Eq. (33)]. Letting $C$ denote the actual concentration in vapor or liquid, we can write

$$
C^{\prime}=\zeta C /(1+\sigma) .
$$

The dimensionless quantity $K$ in Eq. (49) is defined as

$$
K=b^{*}\left(4 \pi \Delta G^{*} / k_{b} T\right)^{-1 / 2}(1+\sigma) \ln (1+\sigma) / \sigma,
$$

in which $b^{*}$ is a dimensionless capture factor. Following Lewis,

$$
b^{*}=2 \pi / K_{0}\left(r^{*} / \lambda_{s}\right) I_{0}\left(r^{*} / \lambda_{s}\right) .
$$

Here $K_{0}$ and $I_{0}$ are the modified Bessel functions of order zero, with the asymptotic behavior $K_{0}\left(r^{*} / \lambda_{s}\right) I_{0}\left(r^{*} / \lambda_{s}\right)$ $\approx \lambda_{s} / 2 r^{*}$, as $r^{*}>\lambda_{s}$. Taking a critical nucleus of circular shape with height $d_{h k l}$, the classical nucleation theory gives

$$
\begin{aligned}
\Delta G^{*} / k_{b} T & =\pi \gamma^{2} /\left[\left(k_{b} T\right)^{2} \sigma\right] \\
& \approx \pi\left(1-\eta_{h k l}\right)^{2} C_{l(h k l)}^{* 2} \varepsilon^{2} /\left(2 n_{h k l} \sigma\right)
\end{aligned}
$$

and

$$
r^{*}=\gamma a /\left(k_{b} T \sigma\right) \approx\left(1-\eta_{h k l}\right) C_{l(h k l)}^{*} \varepsilon a /\left(n_{h k l} \sigma\right) .
$$

For this type of nucleus, $a^{2} \approx \Omega / d_{h k l}$. Therefore

$$
\begin{aligned}
R_{h k l} \approx & 2 \Omega L^{2} C \sigma^{1 / 2} \zeta \ln (1+\sigma)\left(\sigma d_{h k l}\right)^{-1} \\
& \times \exp \left[(1-t) C_{l(h k l)}^{*} \eta_{h k l} \varepsilon / 4\right. \\
& \left.-\pi\left(1-\eta_{h k l}\right)^{2} C_{l(h k l)}^{* 2} \varepsilon^{2} /\left(2 n_{h k l} \sigma\right)\right] .
\end{aligned}
$$

In this mononucleation model, $L^{2}$ has a conservative influence on the habit of crystals. Consider a crystal bounded by faces of the same crystallographic form; all will have roughly the same area. Suppose that due to a temporary fluctuation one face grows fast and its area decreases, leading to a decrease in $R_{h k l}$. Simply assuming that $L^{2}$ is similar for all faces, we obtain

$$
\begin{aligned}
R_{h k l}^{\mathrm{red}} \sim \zeta\left(d_{h k l}\right)^{-1} \exp \left[(1-t) C_{l(h k l)}^{*} \eta_{h k l} \varepsilon / 4\right. \\
\\
\left.-\pi\left(1-\eta_{h k l}\right)^{2} C_{l(h k l)}^{* 2} \varepsilon^{2} /\left(2 n_{h k l} \sigma\right)\right] .
\end{aligned}
$$

Based on Eq. (56), $R_{h k l}^{\mathrm{red}} d_{h k l}$ is plotted versus $\eta_{h k l}$ for different $\sigma\left(t=0.25, n_{h k l}=4, C_{l(h k l)}^{*}=0.5, \varepsilon=6\right)$ in Fig. 5(a). An evident result obtained from this figure is that $R_{h k l}^{\mathrm{red}}$ is nonlinearly dependent on $\eta_{h k l}$ at low supersaturations, while at
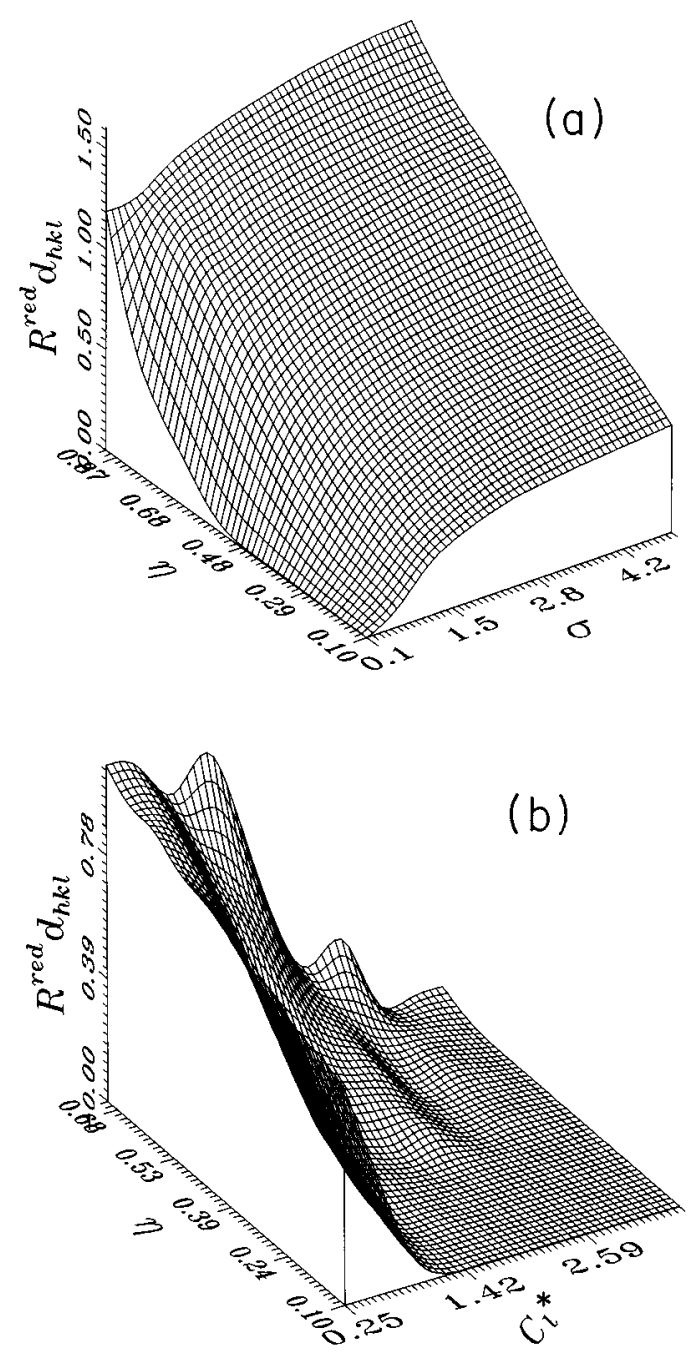

FIG. 5. Reduced growth rate plotted as a function of various parameters. Mononucleation model [Eq. (56)]. (a) $R_{h k l}^{\text {red }} d_{h k l}$ plotted vs $\eta_{h k l}$ for various $\sigma ; t=0.25, n_{h k l}=4, C_{l(h k l)}^{*}=0.5, \varepsilon=6$. (b) $R_{h k l}^{\text {red }} d_{h k l}$ plotted as a function of $\eta_{h k l}$ and $C_{l(h k l)}^{*} ; n_{h k l}=4, \varepsilon=6$, $\sigma=2, t=0.25$.

high supersaturations $R_{h k l}^{\text {red }}$ is roughly proportional to $\eta_{h k l} / d_{h k l}$. This result is very different from the result of the BCF model where at high supersaturations $R_{h k l}^{\mathrm{red}} \sim d_{h k l} \eta_{h k l}$. In Fig. 5(b), $R_{h k l}^{\text {red }} d_{h k l}$ is plotted as a function of $\eta_{h k l}$ and $C_{l(h k l)}^{*}$. Similar to previous cases, $R_{h k l}^{\mathrm{red}} d_{h k l}$ is strongly dependent on $\eta_{h k l}$ and $C_{l(h k l)}^{*}$. It is interesting to see that for those faces with a high $\eta_{h k l}$ the change in $C_{l(h k l)}^{*}$ may cause oscillations of $R_{h k l}^{\text {red }} d_{h k l}$. This probably implies that the growth occurring in that region may lead to the morphological instability.

\section{Birth and spread model}

According to this model, a second nucleus may appear either on top of the first nucleus or elsewhere on the face before the first nucleus has grown out to cover the entire face. For the growth rate, Lewis arrives at 
TABLE I. Habit-controlling factors and reduced growth rates for $F$ faces of $n-\mathrm{C}_{24} \mathrm{H}_{50}$ crystals.

\begin{tabular}{lccccc}
\hline \hline Forms & $n_{h k l}{ }^{\text {a }}$ & $d_{h k l}{ }^{\mathrm{a}}$ & $\eta_{h k l}{ }^{\mathrm{a}}$ & $C_{l(h k l)}^{*}$ & $R_{h k l}^{\mathrm{red}}$ \\
\hline$\{001\}$ & 3 & 30.24 & 0.0509 & 0.993 & 1 \\
$\{010\}$ & 2 & 4.596 & 0.545 & 0.372 & 304 \\
$\{011\}$ & 2.1 & 4.509 & 0.563 & 0.363 & 400 \\
$\{100\}$ & 2.5 & 3.815 & 0.681 & 0.242 & $2.68 \times 10^{3}$ \\
$\{101\}$ & 2.5 & 3.967 & 0.686 & 0.244 & $2.81 \times 10^{3}$ \\
$\{111\}$ & 2.8 & 3.582 & 0.776 & 0.205 & $6.91 \times 10^{3}$ \\
$\{110\}$ & 2.9 & 3.484 & 0.803 & 0.187 & $9.87 \times 10^{3}$ \\
\hline \hline
\end{tabular}

${ }^{\mathrm{a}}$ Selected from Ref. 33.

$$
R_{h k l}=B^{\prime} \exp \left(-\Delta G^{*} / 3 k_{b} T\right)
$$

with

$$
B^{\prime}=(4 K)^{1 / 3} \sigma \zeta C \lambda_{s} /(1+\sigma) .
$$

It is easy to see that one of the main differences between the results of this model and those from the mononucleation model is that the growth rate no longer depends on $L^{2}$. Applying the same technique as in the previous sections, we find

$$
R_{h k l} \approx 2 C \sigma^{5 / 6} \zeta \Omega^{2 / 3}[\ln (1+\sigma) / \sigma]^{1 / 3}\left[(1+\sigma) d_{h k l}\right]^{-2 / 3} \exp \left[(1-t) C_{l(h k l)}^{*} \eta_{h k l} \varepsilon / 4-\pi\left(1-\eta_{h k l}\right)^{2} C_{l(h k l)}^{* 2} \varepsilon^{2} /\left(6 n_{h k l} \sigma\right)\right]
$$

and

$$
R_{h k l}^{\mathrm{red}} \sim \zeta\left(d_{h k l}\right)^{-2 / 3} \exp \left[(1-t) C_{l(h k l)}^{*} \eta_{h k l} \varepsilon / 4-\pi\left(1-\eta_{h k l}\right)^{2} C_{l(h k l)}^{* 2} \varepsilon^{2} /\left(6 n_{h k l} \sigma\right)\right]
$$

Comparison of (56) with (60) reveals that the relative growth rate depends in a different way on the same factors as in the mononucleation model. Therefore similar relations as shown in Fig. 5 can also be obtained for the case of the birth and spread model.

\section{COMPARISON WITH OBSERVED MORPHOLOGY}

In this section, we take crystals of even triclinic alkanes $\left(n-\mathrm{C}_{24} \mathrm{H}_{50}\right)$ and of $\mathrm{C}_{60}$ as an example, to compare the predicted growth forms with the observed growth forms.

According to our experiments and those of other authors, ${ }^{31}$ the screw dislocation mechanism plays an essential role in the case of alkane crystals grown from solutions. For that reason Eq. (45) is applied to predict the morphology of the crystals. Triclinic $n$-paraffin crystals are of the space group symmetry $P_{1} \cdot{ }^{32}$ According to our periodic-bond-chain (PBC) analysis ${ }^{33}$ the crystal faces $\{001\},\{010\},\{011\},\{100\}$, $\{101\},\{111\}$, and $\{110\}$ are important $F$ faces. The parameters and calculated results for $n-\mathrm{C}_{24} \mathrm{H}_{50}$ crystals $(\varepsilon \approx 33.1)$ are listed in Table I. Here $d_{h k l}, n_{h k l}$, and $\eta_{h k l}$ are obtained from the structure data and the PBC analysis. $C_{l(h k l)}^{*}$ is ob- tained from an interfacial structural analysis (IS), based on Eq. (11) and the results of self-consistent-field calculations. $^{25,34}$

$\mathrm{C}_{60}$ crystals have the structure of fcc with four molecules per unit cell. ${ }^{34}$ The crystals normally grow from the vapor $(\varepsilon \approx 23.1)$. (It follows from the PBC analysis ${ }^{9,34}$ and the experiments $^{34}$ that the $\{111\}$ and $\{100\}$ faces of the crystals are morphologically important. Therefore our concentration is focused on these faces.) According to Verheijen et al. ${ }^{35}$ the growth of these crystals is also governed by the screw dislocation mechanism. Therefore, Eq. (35) should be applied in this case. $C_{l(h k l)}^{*}$ is estimated from a moleculardynamic calculation based on Eq. (11). Note that since the growth units are spherical in this case, $t_{h k l}=0$ for all orientations (see the Appendix for more explanations).

Based on the data given in Tables I and II, the theoretical growth forms of $n-\mathrm{C}_{24} \mathrm{H}_{50}$ crystals and $\mathrm{C}_{60}$ crystals are constructed using the Wulff plot. The predicted morphology of $\mathrm{C}_{60}$ crystals is shown in Fig. 6, and the predicted morphology of $n-\mathrm{C}_{24} \mathrm{H}_{50}$ crystals is similar as given in Ref. 25, but much longer in the $b$ direction. For the purpose of comparison, the corresponding theoretical growth forms obtained from

TABLE II. Habit-controlling factors and reduced growth rates for the $\{111\}$ and $\{100\}$ faces of $\mathrm{C}_{60}$ crystals.

\begin{tabular}{lccccccc}
\hline \hline Forms & $n_{h k l}$ & $d_{h k l}$ & $\eta_{h k l}$ & $C_{l(h k l)}^{*}$ & $\sigma_{1}$ & $\zeta$ & $R_{h k l}^{\text {red }}$ \\
\hline$\{111\}$ & 3 & 8.18 & 0.5 & 0.130 & 1.36 & 0.946 & 473 \\
$\{100\}$ & 2 & 7.09 & 0.667 & 0.100 & 0.230 & 0.950 & 539 \\
\hline \hline
\end{tabular}




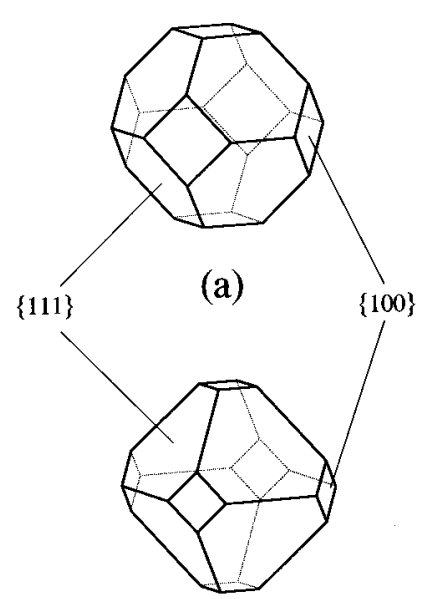

(b)

FIG. 6. Wulff constructions of the growth morphology of $\mathrm{C}_{60}$ crystals grown from the vapor based on (a) our formalism [Eq. (35)] and (b) Hartman-Perdok theory [Eq. (2)].

Hartman-Perdok theory based on Eq. (2) are also presented in these figures. It follows that our formalism predicts long flat needlelike triclinic paraffin crystals, while HartmanPerdok theory predicts platy and somewhat thick crystals (cf. Ref. 25).

It follows from our experiments that triclinic paraffin crystals indeed obtain a long flat needle shape and are bounded by the $\{001\},\{010\}$, and $\{100\}$ faces when they are grown from $n$-hexane or iso-octane (cf. Ref. 25). This is in excellent agreement with our prediction. In contrast, the morphology predicted by Hartman-Perdok theory turns out to be too thick and the crystals are bounded by somewhat different faces (the $\{101\}$ and $\{111\}$ ) in the $a$ direction. In the case of $\mathrm{C}_{60}$ crystals grown from the vapor, both our formalism [Eq. (35)] and Hartman-Perdok theory predict crystals of a truncated octahedral shape bounded by the $\{111\}$ and the $\{100\}$ faces. However, the morphology obtained from our formalism [Fig. 6(a)] reveals larger $\{100\}$ faces than HartmanPerdok theory [Fig. 6(b)]. This is exactly the same as observed experimentally. ${ }^{35}$

\section{DISCUSSION}

\section{A. Validity of Hartman-Perdok theory}

Relation (2) proposed by Hartman and Perdok ${ }^{5-7}$ is widely used to construct growth forms of crystals. Following the discussions in the forgoing sections, it can be seen that $R_{h k l}^{\text {red }}$ is a monotonic function of $\eta_{h k l}$. From this point of view, $\eta_{h k l}$ can only be qualitatively used to rank the morphological importance of the different crystal faces of a crystal. It is not accurate enough to construct growth forms of crystals. Nevertheless, more precise expressions may be roughly simplified to Eq. (2) in some special cases.

Assume that $n_{h k l}$ is the same for all the faces of a crystal and $d_{h k l} \approx\left(1-\eta_{h k l}\right)$. For crystals grown from the vapor, normally $C_{l(h k l)}^{*} \ll 1$. Therefore $\exp \left[(1-t) C_{l(h k l)}^{*} \eta_{h k l} \varepsilon / 4\right] \approx 1 \approx \zeta$. It then follows from Eq. (36) that the relative growth rate can be simplified as

$$
R_{h k l}^{\mathrm{red}} \sim 1 / C_{l(h k l)}^{*}
$$

For systems consisting of structurally simple units, the results obtained from our calculations show that if $\sigma$ is relatively small, $1 / C_{l(h k l)}^{*}$ is a monotonic (or approximately linear) function of $\eta_{h k l}$. This implies that Eq. (36) can be reduced to Eq. (2). From this point of view, Eq. (2) can be to some extent applied to predict growth forms of crystals grown from the vapor.

It has to be noted that the analysis presented above was just to relate the established expressions in Sec. IV with Eq. (2). In most cases, Eq. (2) is still too crude to describe growth forms of crystals. In practice, we propose that, depending on the prevailing conditions, more precise expressions, such as Eqs. (35) [or (36)], (45), etc., should be applied to predict the morphology of crystals.

\section{B. Habit-controlling factors and growth forms}

As seen from the discussions in Sec. III, main habitcontrolling factors can be classified as $d_{h k l}, n_{h k l}, \eta_{h k l}$, $C_{l(h k l)}^{*}, \varepsilon$, and $\sigma$. The growth forms of crystals can be actually considered as the projection of $R_{h k l}^{\text {red }}$ in multipleparameter space of $d_{h k l}, n_{h k l}, \eta_{h k l}, C_{l(h k l)}^{*}, t_{h k l}, \varepsilon$, and $\sigma$. Any change in one of these parameters will have a certain impact on $R_{h k l}^{\mathrm{red}}$ and on the growth habit of crystals. Among these parameters, $d_{h k l}, n_{h k l}$, and $\eta_{h k l}$ are subject to crystallographic orientations and the structure of the crystal surfaces and remain invariant for a given crystal structure. These factors in principle determine the characteristic habit of crystals. From this point of view, they belong to the internal habit-controlling factors. It can be seen from the obtained formalisms in Sec. III that both $n_{h k l}$ and $\eta_{h k l}$ are exponentially related to $R_{h k l}^{\text {red }}$. Regarding the fact that $n_{h k l}$ does not always vary with the orientation, $\eta_{h k l}$ actually becomes a crucial factor of influence on the morphology of crystals in the case where $C_{l(h k l)}^{*}$ is not too small. This has already been illustrated in the figures in Sec. III.

On the other hand, the parameters $C_{l(h k l)}^{*}, t_{h k l}, \varepsilon$, and $\sigma$ are determined mutually by the crystal and ambient phase and by the experimental conditions. From an experimental point of view, these parameters can be altered by changing the experimental conditions or the composition of the ambient phase. Therefore they can be regarded as an external habit-controlling factor.

In many cases it is very desirable to modify the morphology of crystals. This can be fulfilled by changing some of the habit-controlling factors. Following the discussions in Secs. II and III, we notice that $C_{l(h k l)}^{*}$ and $t_{h k l}$ play a very important role in controlling the habit of crystals. Both of them are very sensitive to the change in the solid-fluid structure, which can be easily caused by the solvent or impurities. Therefore one of the most efficient ways to modify the habit of crystals is to change $C_{l(h k l)}^{*}$ and $t_{h k l}$ by introducing tailormade additives to the solution.

The reason that the factors $\eta_{h k l}, C_{l(h k l)}^{*}$, and $t_{h k l}$ are the crucial habit-controlling factors is that they are directly correlated with kinetic processes of crystal growth. First, kinetic factors such as $\lambda_{s}, \beta_{\mathrm{st}}, D_{s}$, etc., are associated with a certain activation energy related to interfacial processes of crystal growth. (See the discussions in Sec. II.) Second, the step energy $\tilde{\phi}_{h k l}^{\text {step }}$ and the step free energy $\gamma$ have a direct link 


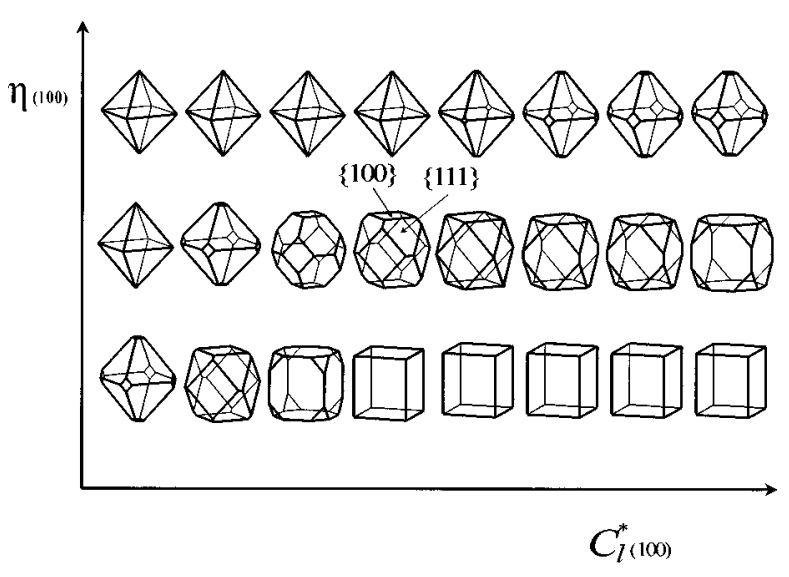

FIG. 7. Schematic illustration of the dependence of the morphology of fcc crystals on $C_{l(h k l)}^{*}$ and $\eta_{h k l}$. Here the $\{100\}$ faces of $\mathrm{C}_{60}$ crystals are taken into account (cf. Table II). $C_{l(h k l)}^{*}$ and $\eta_{h k l}$ from 0.04 to 0.25 and from 0.5 to 0.8 , respectively. With increasing $C_{l(h k l)}^{*}$ or decreasing $\eta_{h k l}$, the relative growth rate of the $\{100\}$ faces decreased, resulting in a large surface area in the orientations. In addition, the morphological change of the crystals reveals the characteristics of nonlinearity.

with $\eta_{h k l}$ and $C_{l(h k l)}^{*}$. The value of the step (free) energy determines the actual height of the two-dimensional nucleation barrier. According to crystal growth theories, ${ }^{1,2}$ to overcome this barrier is one of the rate-determining processes for the growth of flat crystal faces. Hence the role of $\eta_{h k l}$ and $C_{l(h k l)}^{*}$ in the morphology of crystals is rather prominent. Here we take the $\{100\}$ crystal faces of $\mathrm{C}_{60}$ crystals as an example to demonstrate the influence of $\eta_{h k l}$ and $C_{l(h k l)}^{*}$ on the growth morphology of crystals. The dependence of the growth morphology on $\eta_{h k l}$ and $C_{l(h k l)}^{*}$ is illustrated in Fig. 7. It is worthwhile to compare this figure with Fig. 3. As we have already shown in Fig. 3, the relative growth rate will decrease with increasing $C_{l(h k l)}^{*}$ or decreasing $\eta_{h k l}$. Consequently, the $\{100\}$ faces will become larger (see Fig. 7). In spite of this, Fig. 7 shows that the change in the morphology is nonlinear with $\eta_{h k l}$ and $C_{l(h k l)}^{*}$ and both $\eta_{h k l}$ and $C_{l(h k l)}^{*}$ are correlated with each other.

The supersaturation plays a very special role in controlling the habit of crystals. At very low supersaturations, the screw dislocation mechanism is normally operative. In this case, Eqs. (36) and (45) [or (46)] apply, and the habit is independent of the supersaturation. Assume that $n_{h k l}$ and $\varepsilon$ are similar for all cases. It can be seen from Figs. 3 and 4 that $R_{h k l}^{\mathrm{red}} / d_{h k l}$ is dependent very weakly on $\eta_{h k l}$, provided $\eta_{h k l}$ is low and $C_{l(h k l)}^{*}$ and $t_{h k l}$ are high. If competing faces fall in this regime and the differences in $\eta_{h k l}, C_{l(h k l)}^{*}$, and $t_{h k l}$ are not large, the habit may become rather isotropic. However, with increasing $\eta_{h k l}$ (and decreasing $\left.C_{l(h k l)}^{*}\right), R_{h k l}^{\mathrm{red}} / d_{h k l}$ becomes drastically affected by $\eta_{h k l}$ and $C_{l(h k l)}^{*}$. If competing faces have some differences in $\eta_{h k l}$ and $C_{l(h k l)}^{*}$, the crystal habit will be extremely anisotropic. When that a moderate supersaturation is applied, $R_{h k l}^{\text {red }}$ becomes supersaturation dependent. This can be seen from the plots in Fig. 3.

For the screw dislocation mechanism, high supersaturations will result in isotropic growth forms (see Sec. III A).

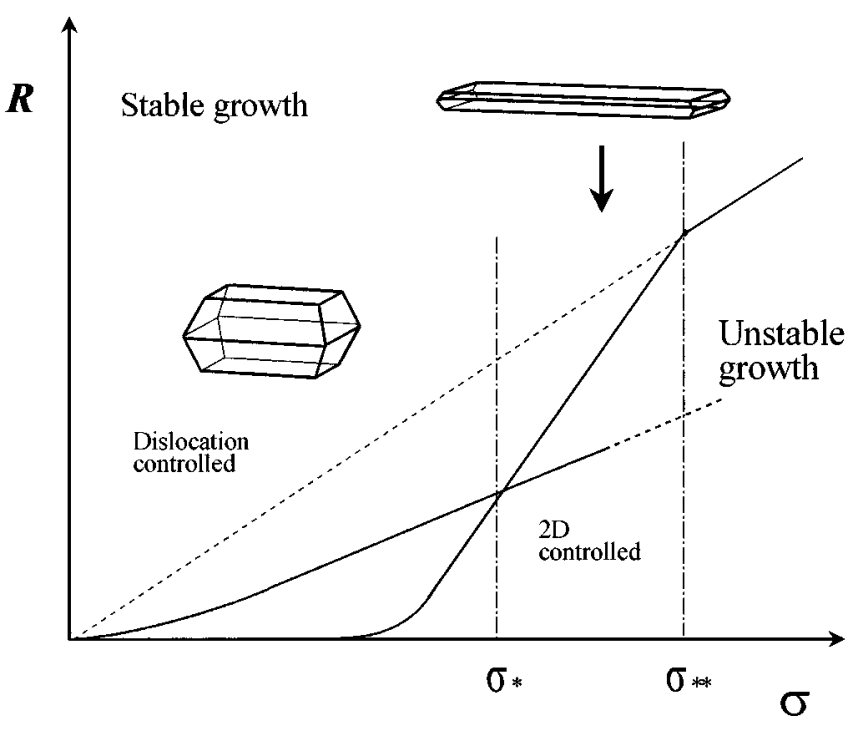

FIG. 8. Dominant growth mechanism and morphology of crystals in different supersaturation regimes. At low supersaturations $\left(\sigma<\sigma^{*}\right)$, the spiral growth mechanism is dominant, whereas at relatively high supersaturations $\left(\sigma^{*}<\sigma<\sigma^{* *}\right)$, the growth of crystals is controlled by the $2 \mathrm{D}$ nucleation mechanism. The $2 \mathrm{D}$-nucleationcontrolled growth will lead to a highly anisotropic growth habit. The morphology shown in this figure is the predicted growth morphology of paraxylene crystals.

However, polynucleation will be the dominant growth mechanism in this case (see Fig. 8). It then follows from Eq. (58) that

$$
R_{h k l}^{\mathrm{red}} \sim \zeta\left(d_{h k l}\right)^{-2 / 3} \exp \left[(1-t) C_{l(h k l)}^{*} \eta_{h k l} \varepsilon / 4\right],
$$

implying that $R_{h k l}^{\text {red }}$ is again supersaturation independent and the growth habit becomes highly anisotropic. The predicted morphological changes of paraxylene crystals in two different supersaturation regimes are illustrated in Fig. 8. Interestingly, this prediction is completely confirmed by the experiments. (The work is in progress.) In addition to these factors, $\varepsilon$ also exerts an influence on $R_{h k l}^{\text {red }}$ (cf. Sec. III A).

We notice that the formalisms obtained in this paper can only be applied to crystals bounded faceted crystals. Under certain conditions, the surface roughening or the surface melting, which have a substantial influence on the growth kinetics and the morphology of crystals, will happen at the flat crystal surfaces. Nevertheless, if some crystal faces roughen either thermally or kinetically, they normally grow much faster than faceted faces and will soon disappear from the morphology. Therefore these rough faces are morphologically unimportant and can be left out of consideration.

In conclusion, the growth habit of crystals is determined by various internal and external controlling factors. Among those, the crystal orientational factor $\eta_{h k l}$, the molecular orientational factors $t_{h k l}$, and the surface scaling factor $C_{l(h k l)}^{*}$ are most crucial. In order to predict the growth morphology of crystals, one should apply a suitable formalism presented in Sec. III, according to the growth mechanism.

\section{ACKNOWLEDGMENTS}

We are very much indebted to Dr. C. S. Strom for her critical reading of this manuscript. We would also like to 
acknowledge Dr. M. A. Verheijen for the interesting discussion of the morphology of $\mathrm{C}_{60}$ crystals and providing some data for our calculations.

\section{APPENDIX}

Before asymmetric growth units can be incorporated into the kinks or the steps at the crystal surfaces, they should adjust themselves in a proper direction or conformation with respect to that of solid units at the surface. The free-energy barrier associated with this process is characterized by the molecular orientational factor $t_{h k l}$. This factor can be approximated as

$$
t_{h k l} \approx\left[\left(\mu^{*}\right)_{A(h k l)}^{\mathrm{eff}}-\left(\mu^{*}\right)_{A(h k l)}\right] / k_{b} T,
$$

where $\left(\mu^{*}\right)_{A(h k l)}^{\text {eff }}$ denotes the standard chemical potential of the effective growth units, while $\left(\mu^{*}\right)_{A(h k l)}$ denotes the average standard chemical potential of growth units at the crystal surface $(h k l)$. According to basic principles of thermodynamics, ${ }^{13}$ the corresponding chemical potentials can be expressed as

$$
(\mu)_{A(h k l)}^{\mathrm{eff}}=\left(\mu^{*}\right)_{A(h k l)}^{\mathrm{eff}}+k_{b} T \ln X_{A(h k l)}^{\mathrm{eff}}
$$

and

$$
(\mu)_{A(h k l)}=\left(\mu^{*}\right)_{A(h k l)}+k_{b} T \ln X_{A(h k l)} .
$$

Considering the equilibrium condition

$$
(\mu)_{A(h k l)}^{\mathrm{eff}}=(\mu)_{A(h k l)},
$$

one immediately obtains, by substituting (A2) and (A3) into (A1),

$$
t_{h k l} \approx-\ln \left[X_{A(h k l)}^{\mathrm{eff}} / X_{A(h k l)}\right] .
$$

Since $X_{A(h k l)}^{\text {eff }} \leqslant X_{A(h k l)}, t_{h k l} \geqslant 0$. Obviously, $t_{h k l}=0$ corresponds to $X_{A(h k l)}^{\text {eff }}=X_{A(h k l)}$, meaning that all growth units at the crystal surface are dynamically equivalent in the growth process. The growth of crystals with spherical structural units is one of the examples. $t_{h k l} \gg 0$ implies that only a very small fraction of growth units at the crystal surface has the proper orientation or conformation with respect to the solid units, and the conformational barrier for other growth units to transform into the effective growth units is very high. The growth of polyethylene crystals in the $\{001\}$ orientations belongs to this case.
${ }^{1}$ P. Bennema and J. P. van der Eerden, in Morphology of Crystals, edited by I. Sunagawa (Terra Science, Tokyo, 1987), Pt. A, p. 1.

${ }^{2}$ P. Bennema, in Handbook on Crystal Growth, edited by D. T. J. Hurle (North-Holland, Amsterdam, 1993), p. 477.

${ }^{3}$ N. Steno, The Prodromus of Nicolaus Steno's Dissertation Concerning a Solid Body Enclosed by Process of Nature Within a Solid, English translation by J. G. Winter (Hofner, New York, 1968).

${ }^{4}$ J. D. H. Donnay and D. Harker, Am. Mineral. 22, 446 (1937).

${ }^{5}$ P. Hartman and W. G. Perdok, Acta Crystallogr. 8, 49 (1955).

${ }^{6}$ P. Hartman and W. G. Perdok, Acta Crystallogr. 8, 521 (1955).

${ }^{7}$ P. Hartman and W. G. Perdok, Acta Crystallogr. 8, 145 (1955).

${ }^{8}$ P. Hartman and P. Bennema, J. Cryst. Growth 49, 145 (1980).

${ }^{9}$ P. Hartman, in Morphology of Crystals (Ref. 1), p. 271.

${ }^{10}$ P. Bennema, X. Y. Liu, K. Lewtas, R. D. Tack, J. J. M. Rijpkema, and K. J. Roberts, J. Cryst. Growth 121, 679 (1992).

${ }^{11}$ X. Y. Liu and P. Bennema, J. Appl. Crystallogr. 26, 229 (1993).

${ }^{12}$ X. Y. Liu and P. Bennema, J. Chem. Phys. 97, 3600 (1992).

${ }^{13} \mathrm{~S}$. R. Fowler and E. A. Guggenhein, Statistical Thermodynamics (Cambridge University Press, London, 1960).

${ }^{14}$ X. Y. Liu and P. Bennema, J. Chem. Phys. 98, 5863 (1993).

${ }^{15}$ K. A. Jackson, Liquid Metals and Solidification (American Society for Metals, Metal Park, OH, 1958).

${ }^{16}$ J. Q. Broughton and F. F. Abraham, Chem. Phys. Lett. 71, 456 (1980).

${ }^{17}$ J. Q. Broughton and G. H. Gilmer, J. Chem. Phys. 79, 5090 (1983); 79, 5105 (1983); 79, 5119 (1983); 84, 5741 (1986); 84, 5749 (1986); 84, 5759 (1986).
${ }^{18}$ A. Bonissent, in Interfacial Aspects of Phase Transformation, edited by B. Mutaftschiev (Reidel, Dordrecht, 1982).

${ }^{19}$ A. D. J. Haymet and D. W. Oxtoby, J. Chem. Phys. 74, 2559 (1981).

${ }^{20}$ W. A. Curtin and N. W. Ascroft, Phys. Rev. A 32, 2909 (1985).

${ }^{21}$ X. Y. Liu, Surf. Sci. 290, 403 (1993).

${ }^{22}$ X. Y. Liu, J. Chem. Phys. 98, 8154 (1993).

${ }^{23}$ C. Herring, Phys. Rev. 82, 87 (1951).

${ }^{24}$ C. Rottman and M. Wortis, Phys. Rep. 103, 59 (1984).

${ }^{25}$ X. Y. Liu and P. Bennema, Phys. Rev. B 49, 765 (1994).

${ }^{26}$ X. Y. Liu (unpublished).

${ }^{27}$ X. Y. Liu, E. S. Boek, W. J. Briels, and P. Bennema, Nature 374, 342 (1995).

${ }^{28}$ A. A. Chernov, Modern Crystallography III-Crystal Growth (Springer-Verlag, Berlin, 1984).

${ }^{29}$ P. Bennema and G. H. Gilmer, in Crystal Growth: An Introduction, edited by P. Hartman (North-Holland, Amsterdam, 1973), p. 263.

${ }^{30}$ B. Lewis, J. Cryst. Growth 21, 29 (1974).

${ }^{31} \mathrm{R}$. Boistelle, in Current Topics in Materials Science, edited by E. Kaldis (North-Holland, Amsterdam, 1980), p. 413.

${ }^{32}$ S. C. Nyburg and J. A. Potworowski, Acta Crystallogr. B 29, 347 (1973).

${ }^{33}$ X. Y. Liu and P. Bennema, J. Cryst. Growth 135, 209 (1994).

${ }^{34}$ X. Y. Liu and P. Bennema, Phys. Rev. E 48, 2006 (1994).

${ }^{35}$ M. A. Verheijen, H. Meekes, G. Meijer, E. Raas, and P. Bennema, Chem. Phys. Lett. 191, 339 (1992). 